10 years ESJ

Special edition

\title{
Preface to Computational Intelligence Applications in Medicine and Biology
}

\author{
Daniel B. Hier, MD \\ Donald C. Wunsch II, PhD \\ Applied Computational Intelligence Laboratory \\ Missouri University of Science and Technology
}

Doi:10.19044/esj.2022.v18n4p1

Published: 08 February 2022

Copyright 2022 Author(s)

Under Creative Commons BY-NC-ND

4.0 OPEN ACCESS

Cite As:

Hier D.B. \& Wunsch D.C. (2022). Preface to Computational Intelligence Applications in Medicine and Biology. European Scientific Journal, ESJ, 18 (4), 1. https://doi.org/10.19044/esj.2022.v18n4p1

This special edition of the European Science Journal is devoted to applying computational intelligence methods to solving complex problems in medicine and biology. Several significant trends have fostered progress in this area that include:

Mathematical models of complex biological phenomena are increasingly available (Smye \& Clayton, 2002).

Large disease datasets with hundreds or thousands of patient entries are publicly available (Purushotham, Meng, Che, \& Liu, 2018; Obermeyer \& Emanuel, 2016).

Supervised machine learning, unsupervised machine learning, and deep learning algorithms are being successfully applied to solve problems in biology and medicine (Al-Jabery, Obafemi-Ajayi, Olbricht, \& Wunsch, 2019; Xu \& Wunsch, 2010; Liu, Chen, Krause, \& Peng, 2019)

Advances in natural language processing and ontology development are transforming previously impenetrable warehouses of unstructured text data into computable data sources (Fu et al., 2020; Gehrmann et al., 2018; Yang, Bian, Hogan, \& Wu, 2020). 
This special edition contains five contributions from the Applied Computational Intelligence Laboratory (ACIL) at the Missouri University of Science and Technology in Rolla, MO, USA.

\section{A Focused Review of Deep Phenotyping with Examples from Neurology}

by Hier et al. introduces the concept of what is a patient phenotype and demonstrates how vectorization of phenotypic features can make patient phenotype computable. Patient phenotypes are signs and symptoms of disease that permit the characterization of diseases and their subtypes. Patient phenotypes can be correlated with disease outcomes and response to treatment as part of precision medicine initiatives. The concepts needed for patient phenotyping are derived from large online ontologies with machine readable codes.

\section{Subsumption is a Novel Feature Reduction Strategy for High Dimensionality Datasets}

by Wunsch \& Hier argues that when phenotypic features are organized in a hierarchical ontology, subsumption can be an effective strategy for reducing high dimension clinical datasets.

\section{High Throughput Phenotyping of Neurological Patients with MetaMap}

by Hier et al. explores the utility of this powerful natural language processing tool to extract concepts from the medical text. MetaMap is able to extract phenotypic concepts from electronic health records or online disease summaries with and accuracy of $61-89 \%$.

\section{A Robot Advisor to Improve Computerized Game Play}

explores whether a robot advisor can improve computerized gameplay. Robot advisors are showing increasing use in healthcare. The study of Tanksley et al. examines whether a robot advisor can improve computerized game play and whether carryover learning occurs when the robot advisor is not active. This study has implications for improving health outcomes through gamification strategies (Sardi, Idri, \& Fernandez- Aleman, 2017).

Reproducing Neural Network Research Findings via Reverse Engineering: Replication of AlphaGo Zero by Crowdsourced Leela Zero examines reproducibility issues of deep learning algorithms. Tanksley et al. show how crowdsourcing was able to replicate the remarkable accomplishment of DeepMind who constructed a neural network that became the best Go player in the world. This issue is especially relevant to neural network models in healthcare (Beam, Manrai, \& Ghassemi, 2020). 


\section{Conflicts of Interest}

This Special Edition was initiated at the invitation of the Editorial Office of the European Scientific Journal. All submissions were peerreviewed by external reviewers. The Editors of this Special Edition have no conflicts of interest to disclose.

\section{References:}

1. Al-Jabery, K., Obafemi-Ajayi, T., Olbricht, G., \& Wunsch, D. (2019). Computational learning approaches to data analytics in biomedical applications. Academic Press.

2. Beam, A. L., Manrai, A. K., \& Ghassemi, M. (2020). Challenges to the reproducibility of machine learning models in health care. Jama, 323 (4), 305-306.

3. Fu, S., Chen, D., He, H., Liu, S., Moon, S., Peterson, K. J., and others (2020). Clinical concept extraction: a methodology review. Journal of Biomedical Informatics, 103526.

4. Gehrmann, S., Dernoncourt, F., Li, Y., Carlson, E. T., Wu, J. T., Welt, J., and others (2018). Comparing deep learning and concept extractionbased methods for patient phenotyping from clinical narratives. PloS one, 13 (2), $\mathrm{e} 0192360$.

5. Liu, Y., Chen, P.-H. C., Krause, J., \& Peng, L. (2019). How to read articles that use machine learning: users' guides to the medical literature. JAMA, 322 (18), 1806-1816.

6. Obermeyer, Z., \& Emanuel, E. J. (2016). Predicting the future-big data, machine learning, and clinical medicine. The New England Journal of Medicine, 375 (13), 1216.

7. Purushotham, S., Meng, C., Che, Z., \& Liu, Y. (2018). Benchmarking deep learning models on large healthcare datasets. Journal of Biomedical Informatics, 83, 112-134.

8. Sardi, L., Idri, A., \& Fern andez-Alemªn, J. L. (2017). A systematic review of gamification in e-health. Journal Of Biomedical Informatics, 71, 31-48. Smye, S. W., \& Clayton, R. H. (2002). Mathematical modeling for the new millennium: medicine by numbers. Medical Engineering \& Physics, 24 (9), 565-574.

9. Xu, R., \& Wunsch, D. C. (2010). Clustering algorithms in biomedical research: A review. IEEE Reviews in Biomedical Engineering, 3, 120154. doi: 10.1109/RBME.2010.2083647

10. Yang, X., Bian, J., Hogan, W. R., \& Wu, Y. (2020). Clinical concept extraction using transformers. Journal of the American Medical Informatics Association, 27 (12), 1935-1942. 